\title{
Hyperfragments from the Lightest p-shell Hypernuclei. 2. Recent Progress and the Next Steps
}

\author{
Olga Majlingová*, ${ }^{\dagger}$ \\ Czech Technical University, Prague, Czech Republic \\ E-mail: olga.majlingova@fs.cvut.cz
}

\section{Lubomir Majling,}

Nuclear Physics Institute, Řežnear Prague, Czech Republic and Bogoliubov Laboratory of Theoretical Physics, JINR, Dubna, Russia E-mail: majling@ujf.cas.cz

\section{Petr Bydžovský,}

Nuclear Physics Institute, Řež near Prague, Czech Republic

E-mail: bydzovsky@ujf.cas.cz

\section{Patrick Achenbach}

Institut für Kernphysik, J. Gutenberg-Universität Mainz, Germany

E-mail: patrick@kph.uni-mainz.de

Background: The extension of the shell model for hypernuclei suggested by Gal and Millener is very successfully used in the interpretation of the discrete part of the spectra of $p$-shell hypernuclei. Recently, the identification of ${ }_{\Lambda}^{4} \mathrm{H}$ hyperfragments from a ${ }^{9} \mathrm{Be}$ target was confirmed in the fragmentation experiment at MAMI.

Purpose: The advanced study of the strong decay of primary $p$-shell hypernuclei produced in the reaction ${ }^{A} Z\left(e, e^{\prime} K^{+}\right)_{\Lambda}^{A}(Z-1)$ provides options in determining and interpreting a still unexplored region of these spectra.

Method: We extend the Translation Invariant Shell Model by including the next excitation $\left(\mathrm{N}_{\min }+2\right)$ and calculate 3-particle coefficients of parentage.

Result: We suggest suitable targets and possible hyperfragments to be registered in fragmentation experiments.

XXII International Baldin Seminar on High Energy Physics Problems

15 - 20 September, 2014

JINR, Dubna, Russia

*Speaker.

${ }^{\dagger}$ Work of L.M.and P.B. was supported by grant P203/12/2126 of the Grant Agency of the Czech Republic. It is also part of the European Union Initiative FP7, Study of Strongly Interacting Matter (HadronPhysics3, SPHERE network). Work of O.M. was supported by Project LG14004 of the Ministry of Education, Youth and Sport of the Czech Republic. 


\section{Introduction}

The study of hypernuclei is of growing importance for contemporary physics. Therefore new results on the hypernuclei are also traditionally presented and discussed in every Baldin seminar, which centers rather on the high-energy physics. Let us recall the session in 1990, when the data obtained by Khorozov and Lukstins were lively discussed. At this meeting the possibility to detect hypernuclei in the BM@N and MPD/NICA detectors was presented [1].

Investigation of production and properties of the baryonic systems with strangeness is also the subject of the European project SPHERE (Strange Particles in Hadronic Environment Research in Europe) on which we have reported in our previous contribution [2]. In this frame properties of hyperfragments are intensively studied both experimentally [3] and theoretically.

In this paper we continue our discussion of possibilities provided by decay-pion spectroscopy $[3,4]$. The pioneering experiment performed at MAMI succeeded in the identification of the ${ }_{\Lambda}^{4} \mathrm{H}$ hyperfragment in electroproduction off a ${ }^{9} \mathrm{Be}$ target proving the feasibility of the method. The method can be used in studying the light hyperfragments which decay dominantly via the two-body pionic decay. One important motivation to study hypernuclei is the possible difference between the hyperon-proton and hyperon-neutron interaction. These experiments allow us to get information not only about a breaking of charge symmetry but also about the cluster structure of light nuclei and, hopefully, about the existence of predicted heavy hyperhydrogen isotopes [5].

\section{Shell Model}

Extensive studies of hypernucleus production have been made using the missing-mass spectroscopy in meson or electron induced reactions

$$
{ }^{A+1} \mathrm{Z}(a, b){ }^{A+1} \mathrm{Z}^{\prime},
$$

where $(a, b)=(K, \pi)$, or $(\pi, K)$, or $\left(e, e^{\prime} K^{+}\right)$[6]. The hypernucleus is produced in a nucleon-hole $\Lambda$-particle state when a nucleon in the target nucleus in a state $j_{n}$ is converted to the $\Lambda$ hyperon in a state $\ell$ :

$$
\mid\left(j_{n}^{-1} \ell_{\Lambda}\right)^{\Delta J} \otimes \Psi^{A}>\quad \ell=0 s, 1 p, 2 d, 2 s, \cdots,
$$

where $\Psi^{A}$ stands for the wave function of the ground state of the core nucleus. This description obvious in closed-shell nuclei can be used for all $p$-shell hypernuclei [2]. 
In Fig. 1 one can see a clear structure in the production spectrum of the ${ }_{\Lambda}^{16} \mathrm{~N}$ hypernucleus [7] which corresponds to the $p_{\frac{1}{2}}^{-1} s_{\Lambda}, p_{\frac{3}{2}}^{-1} s_{\Lambda}, p_{\frac{1}{2}}^{-1} p_{\Lambda}$, and $p_{\frac{3}{2}}^{-1} p_{\Lambda}$ states.

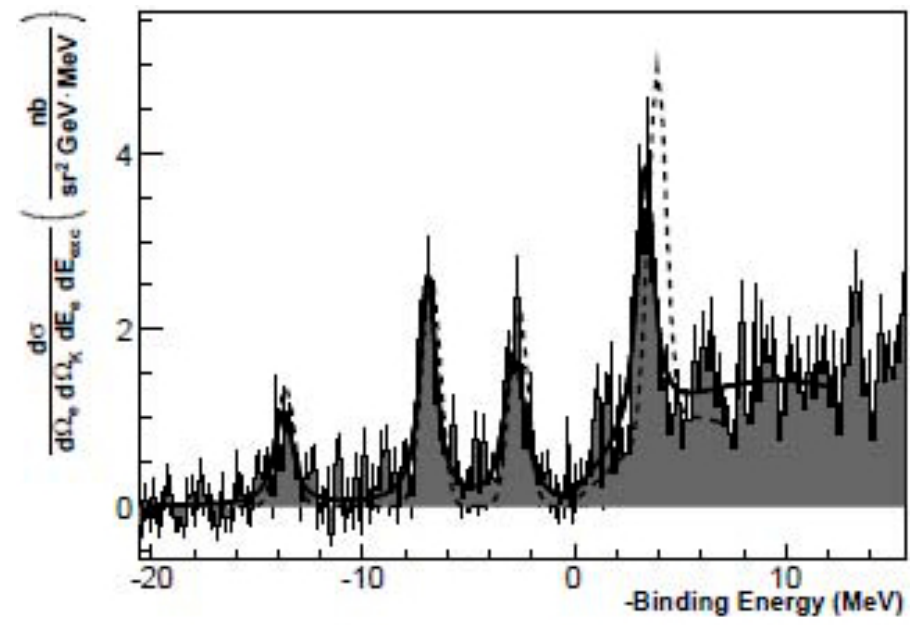

Figure 1: Excitation spectrum of ${ }_{\Lambda}^{16} \mathrm{~N}$ from electroproduction.

A general method for shell model analysis was set up in seminal papers by Gal, Soper and Dalitz [8] introducing the notion of "weak coupling" scheme in which a $\Lambda$ single-particle state is coupled to the wave function of the core nucleus

$$
\Psi\left({ }_{\Lambda}^{A+1} Z\right)=\Psi\left({ }^{A} Z\right) \otimes \psi^{\Lambda} .
$$

For $p$-shell hypernuclei this reads as

$$
\Psi\left({ }_{\Lambda}^{A+1} Z\right)=\mid s^{4} p^{A-4}: J_{A}^{\pi} T_{A} \otimes s_{\Lambda}: J>,
$$

where $\mid s^{4} p^{A-4}: J_{A}^{\pi} T_{A}>$ are eigen states of the $p$-shell Hamiltonian for $A$ nucleons. A comprehensive shell model analysis of discrete part of the spectra below the particle-emission threshold (electromagnetic transitions) was performed by Millener [9].

The first $\left(K^{-}, \pi^{-}\right)$experiments [10] populated mainly $\Delta J=0$ states which inspired Lipkin to introduce the notion of Strangeness Analogue States [11]. We have discussed [12, 13, 14] peculiarities of the decay of the highly excited $s_{n}^{-1} s_{\Lambda}$ state: a narrow width in ${ }_{\Lambda}^{6} \mathrm{Li}$, and various selection rules [2]. Production of the $A=4$ hypernuclei ${ }_{\Lambda}^{4} \mathrm{He}$ and ${ }_{\Lambda}^{4} \mathrm{H}$ is expected.

Our interest in strong decays of excited hypernuclear states is revived due to the successful experiment done by A1 Collaboration at MAMI [4]. In this experiment, the hyperfragment ${ }_{\Lambda}^{4} \mathrm{H}$ was clearly identified in the reaction

$$
\begin{array}{r}
{ }^{9} \mathrm{Be}\left(e, e^{\prime} K^{+}\right){ }_{\Lambda}^{9} \mathrm{Li}^{*} \rightarrow X+{ }_{\Lambda}^{4} \mathrm{H} \\
{ }_{\Lambda}^{4} \mathrm{H} \rightarrow{ }^{4} \mathrm{He}+\pi^{-} .
\end{array}
$$

In the MAMI experiment a large fraction of produced hyperfragments were stopped inside the target before the decay. Two-body pionic decays of these hyperfragments resulted in monoenergetic pions. By measuring the pion momenta using high resolution magnetic spectrometers 
one gains direct access to the ground-state masses of the hyperfragments. From the first experiment the $\Lambda$ separation energy of ${ }_{\Lambda}^{4} \mathrm{H}$ was determined.

However, the excitation spectra of primary hypernucleus ${ }_{\Lambda}^{9} \mathrm{Li}$ in this production reaction is without any structure due to a small selectivity: large momentum and spin flip, see Fig. 2 from [15].
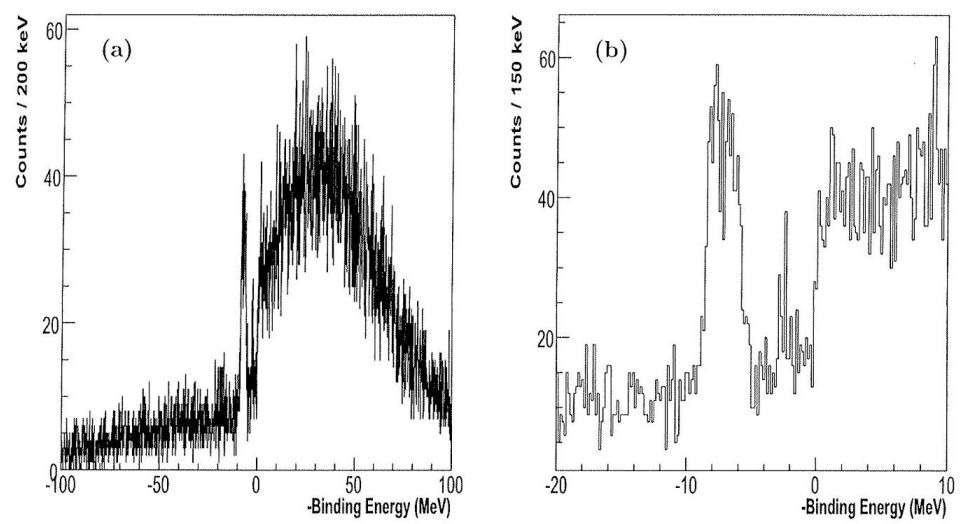

Figure 2: Excitation spectrum of ${ }_{\Lambda}^{9} \mathrm{Li}$ : the whole energy range (a) and the region of interest (b).

It is a great challenge to investigate systematically the production of hyperfragments from primary hypernuclei. In Table 1 we list all possible hyperfragments from light $p$-shell targets. Some of the listed hyperfragments cannot be identified in the MAMI setup: ${ }_{\Lambda}^{4} \mathrm{He}$ decays by $\pi^{0}$ emission and ${ }_{\Lambda}^{5} \mathrm{H}$, if it exists, decays in the three-particle mode, ${ }_{\Lambda}^{5} \mathrm{H} \rightarrow{ }^{4} \mathrm{He}+n+\pi^{-}$.

Table 1: Possible hyperfragments from light $p$-shell target nuclei ${ }^{9} \mathrm{Be},{ }^{7} \mathrm{Li}$ and ${ }^{6} \mathrm{Li}$. Values of pion momenta for two-body decays (in Mev/c) are shown right to the hyperisotope name. Threshold energies (in MeV) are shown left to the break-up channel. ${ }_{\Lambda}^{4} \mathrm{He}$ and ${ }_{\Lambda}^{5} \mathrm{H}$ cannot decay by two-body mode. The heavy hyperhydrogen isotopes ${ }_{\Lambda}^{5} \mathrm{H}$ and ${ }_{\Lambda}^{7} \mathrm{H}$ shown in brackets are not known to be bound.

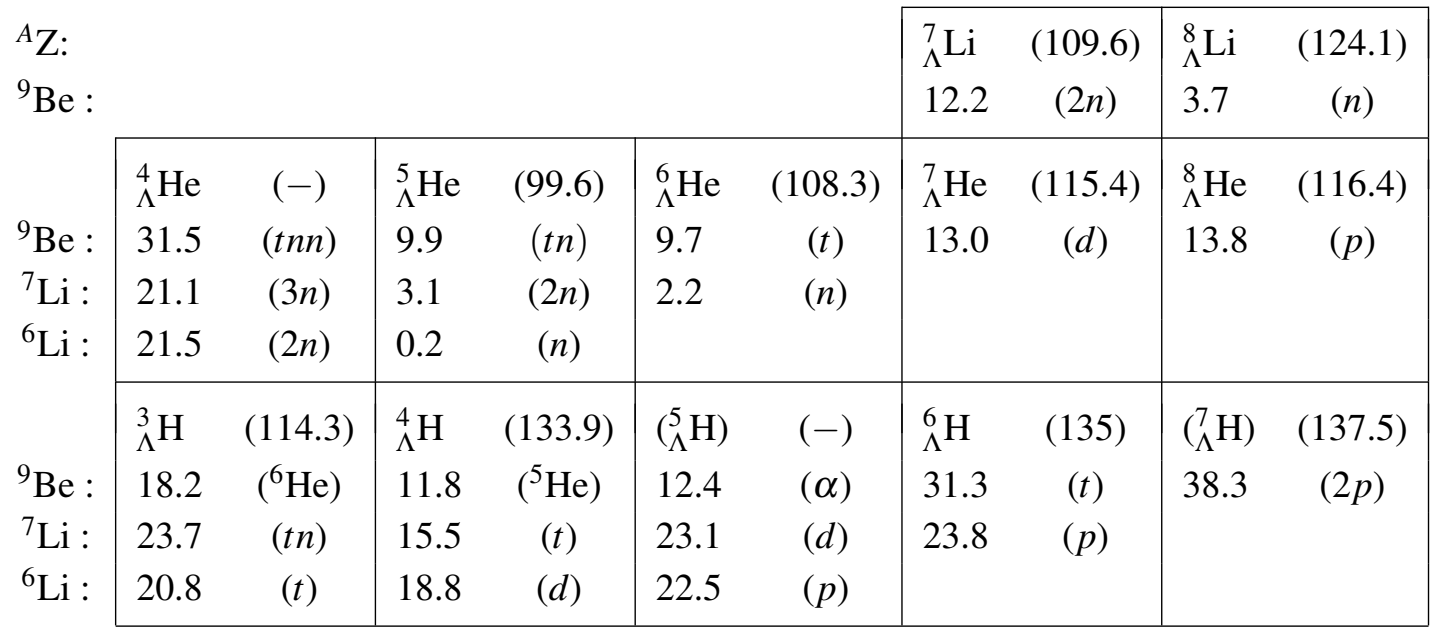




\section{Extended TISM}

We extend the Translation Invariant Shell Model (TISM) [13] by including the next harmonic oscillator excitations $\left(\mathrm{N}_{\min }+2\right)$. We start with the shell model in the LS coupling base. For the light $p$-shell nuclei, this is a surprisingly good approximation, even for modern interactions, see [16]. The antisymmetrized wave function for $k$ particles in the LS base reads as

$$
\left|p^{k}:[f] L S T>=\right| p^{k}:[f](\lambda \mu) L>\cdot \mid \tau^{k}:[\tilde{f}] S T>\quad\left(\tau=\frac{1}{2} \frac{1}{2}\right),
$$

where the Young diagram $[f]=\left[f_{1} f_{2} f_{3}\right]$ labels representation of permutation group with $f_{1} \geq f_{2} \geq f_{3}$ and $f_{1}+f_{2}+f_{3}=k$. This characterizes symmetry of the orbital and spin-isospin parts of the wave function.

Let us also recall the decomposition of the wave function for one particle coefficient of fractional parentage (cfp):

$$
\left|p^{k}:[f](\lambda \mu) L S T>=\sum \sqrt{\frac{n_{f^{\prime}}}{n_{f}}} g_{\ell} g_{S T} \cdot\right| p^{k-1}:\left[f^{\prime}\right](\lambda \mu)^{\prime} L^{\prime} S^{\prime} T^{\prime}>\cdot \mid p>\text {. }
$$

Here, the orbital part of cfp

$$
g_{\ell} \equiv<p^{k-1}(\lambda \mu)^{\prime} L^{\prime} ; p \mid p^{k}(\lambda \mu) L>=\left(\begin{array}{cc|c}
(\lambda \mu)^{\prime} & (10) & (\lambda \mu) \\
L^{\prime} & l=1 & L
\end{array}\right),
$$

is the Clebsch-Gordan Coefficient (CGC) for the group SU(3) [17] and the spin-isospin part of cfp

$$
g_{S T} \equiv<\tau^{k-1}\left[\tilde{f}^{\prime}\right] S^{\prime} T^{\prime} ; \tau \mid \tau^{k}[\tilde{f}] S T>=\left(\begin{array}{cc|c}
{\left[\tilde{f}^{\prime}\right]} & {[\tilde{1}]} & {[\tilde{f}]} \\
S^{\prime} T^{\prime} & \frac{1}{2} \frac{1}{2} & S T
\end{array}\right),
$$

is the CGC for the group SU(4). The weight factor $\frac{n_{f^{\prime}}}{n_{f}}$ in Eq.(3.2) is the ratio of dimensions of representations of the symmetry group $S_{k-1}$ and $S_{k}$.

\subsection{Excited Nuclear States}

For states with several open shells we have to fix the wave function of center-of-mass, $\Psi_{n}\left(R_{A}\right)$. We construct the TISM wave function with total Young scheme $\left[f_{A}\right][18]$

$$
\mid 0 s^{k s}\left[f_{s}\right] ; 1 p^{k p}\left[f_{p}\right](\lambda \mu)_{p} ; \ell^{k \ell}\left[f_{\ell}\right](\lambda \mu)_{\ell}:\left[f_{A}\right](\lambda \mu)_{A} L S T: J>=\sum_{n=0}^{A-4} \Phi_{N}^{(A)}\left[f_{A}\right](\lambda \mu)_{N} \cdot \Psi_{n}\left(R_{A}\right),
$$

where $\ell=2 d, 2 s ; \quad 3 f, 3 p ; \ldots$ with obvious constraints $k s+k p+k \ell=A,\left[f_{s}\right] \otimes\left[f_{p}\right] \otimes\left[f_{\ell}\right]=\left[f_{A}\right]$, and $(\lambda \mu)_{N} \otimes(n 0)=(\lambda \mu)_{A}$.

The unitary transformation from the standard shell model to TISM is performed using the TalmiMoshinsky coefficients. More details can be found in [19]. 
Table 2 shows structure of excited states of $p$-shell nuclei $\Phi_{N}^{(A)}\left[f_{A}\right](\lambda \mu)_{N} \cdot \Psi_{n}\left(R_{A}\right)$ assuming various model spaces. Note that some excited states contain configurations with a "destroyed" Young scheme, i.e. the scheme with $f_{1}<4$ e.g. $\left[f_{A}\right]=[3 \cdots]$ among the " $1 \hbar \omega$ " states and $\left[f_{A}\right]=[2 \cdots]$ among the " $2 \hbar \omega$ " ones.

Table 2: Structure of excited states of $p$-shell nuclei within the frame of the $0 \hbar \omega, 1 \hbar \omega$, and $2 \hbar \omega$ model spaces.

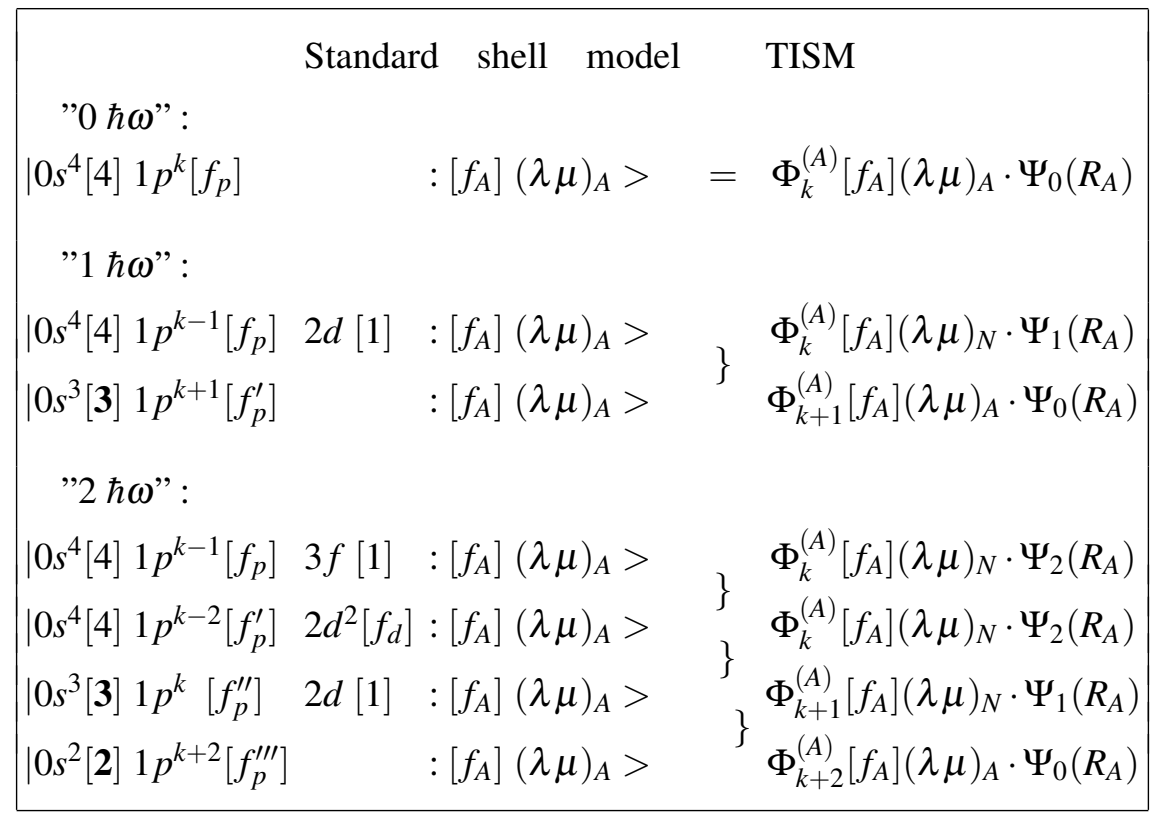

The TISM wave functions for hypernuclei are also constructed using Talmi Moshinsky transformation, see Table $3 \quad\left(\alpha=\sqrt{\frac{A}{A+\mu}}, \quad \beta=\sqrt{\frac{\mu}{A+\mu}}, \quad \mu=\frac{m_{\Lambda}}{m}\right)$. However, if we treat $\Lambda$ as the "last" particle with Jacobi coordinate $\rho=R_{A}-r_{\Lambda}$, then we can write immediately:

$$
\Phi_{N}^{(A)}\left[f_{A}\right](\lambda \mu)_{N} \otimes \varphi_{l}^{\Lambda}(\rho) \quad(\lambda \mu)=(\lambda \mu)_{N} \otimes(l 0)
$$

The structure of excited states of hypernuclei is presented in Table 3.

The list of basis configurations $\Phi_{N}^{(A-1)}\left[f_{A-1}\right] \otimes \varphi_{\ell}^{\Lambda}:\left[f_{A-1}\right](\lambda \mu)_{A}$ is presented in Appendix, Tables 6-9.

\subsection{Coefficients of Fractional Parentage}

The key ingredient in the shell model calculations are coefficients of fractional parentage, coefficients of decomposition of antisymmetric wave function for $A$ particles on product of antisymmetric wave functions for $A_{1}$ and $A_{2}$ particles $\left(A_{1}+A_{2}=A\right)$ and wave function of their relative motion $\varphi_{v}(r)\left(r=R_{A_{1}}-R_{A_{2}}\right)$. 
Table 3: Structure of excited states of $p$-shell hypernuclei.

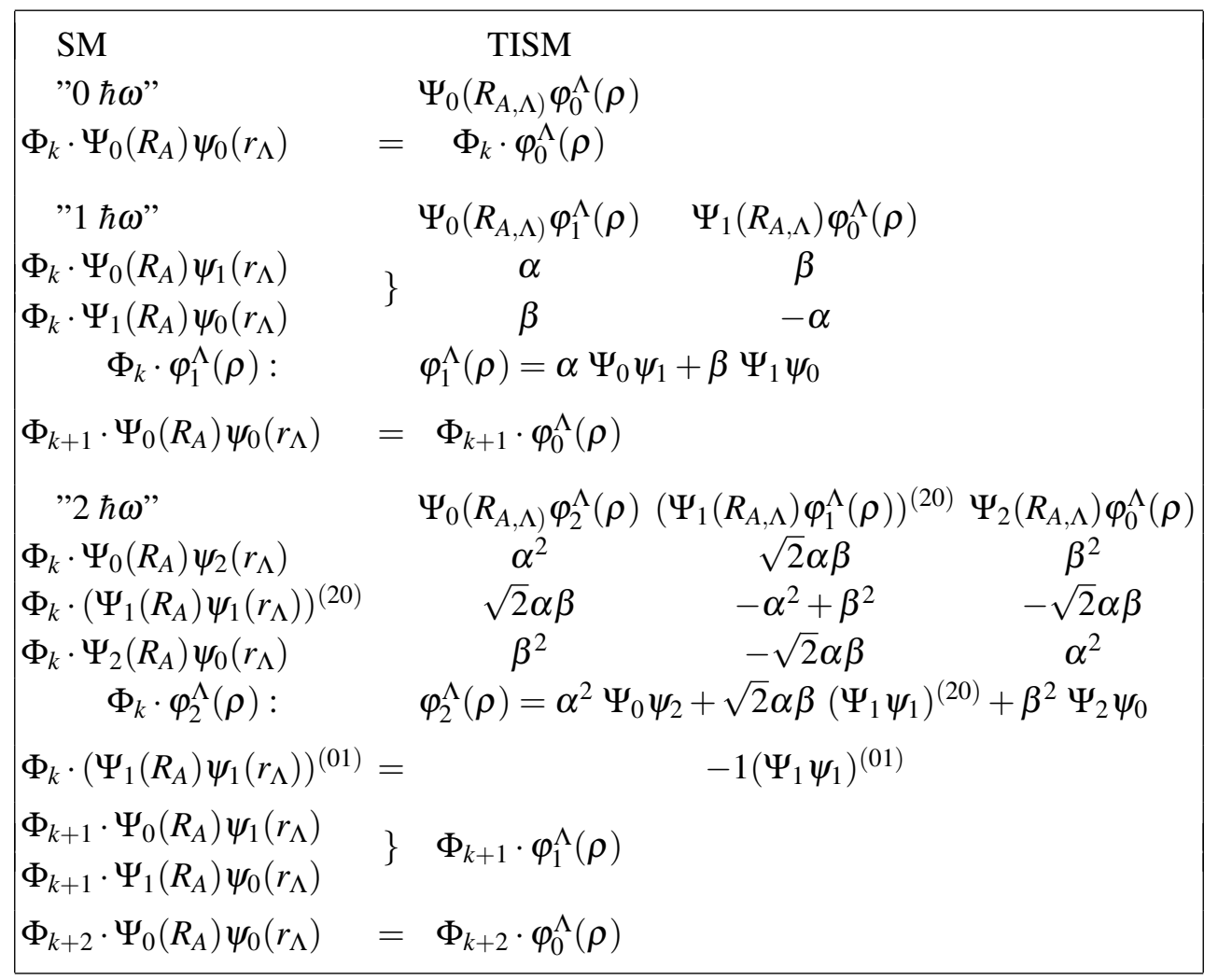

For TISM (harmonic oscillator) wave function we have

$$
\Phi_{N}^{(A)}[f](\lambda \mu)=\sum \sqrt{\frac{n_{f 1} n_{f 2}}{n_{f}}} \cdot G_{L} \cdot G_{S T} \cdot \Phi_{N_{1}}^{\left(A_{1}\right)}\left[f_{1}\right](\lambda \mu)_{1} \cdot \Phi_{N_{2}}^{\left(A_{2}\right)}\left[f_{2}\right](\lambda \mu)_{2} \cdot \varphi_{v}(r),
$$

with constraints $N=N_{1}+N_{2}+v$.

Here, spin-isospin cfp,

$$
G_{S T} \equiv<\tau^{A_{1}}\left[\tilde{f}_{1}\right] S_{1} T_{1} ; \tau^{A_{2}}\left[\tilde{f}_{2}\right] S_{2} T_{2} \mid \tau^{A}[\tilde{f}] S T>=\left(\begin{array}{cc|c}
{\left[\tilde{f}_{1}\right]} & {\left[\tilde{f}_{2}\right]} & {[\tilde{f}]} \\
S_{1} T_{1} & S_{2} T_{2} & S T
\end{array}\right),
$$

is again CGC for group $\mathrm{SU}(4)$.

Orbital cfp

$$
G_{L} \equiv<l^{A_{1}}(\lambda \mu)_{1} L_{1} ; l^{A_{2}}(\lambda \mu)_{2} L_{2} \mid l^{A}(\lambda \mu) L>^{v}
$$

is Racah coefficient (product of four CGC) that describes recoupling of three representations

$$
(\lambda \mu)_{1} \otimes(\lambda \mu)_{2} \otimes(v 0)=(\lambda \mu)
$$

For nucleon clusters, $\mid s^{k}[k](00)>, G_{L}$ is simple CGC: $\quad\left(\begin{array}{cc|c}(\lambda \mu)_{1} & (v 0) & (\lambda \mu) \\ L_{1} & l & L\end{array}\right)$.

The weight factor $\frac{n_{f 1} n_{f 2}}{n_{f}}$ is the ratio of dimensions of representations of the symmetry groups $S_{A_{1}}$, $\mathrm{S}_{\mathrm{A}_{2}}$ and $\mathrm{S}_{A}$. More details are given in [19]. 


\section{Results}

A complete analysis of strong decays is very difficult. To begin with, we demonstrate consequences from the underlying permutation symmetry $[f]_{A}$ for decay $\mathrm{A} \rightarrow \mathrm{A}^{\prime}+\mathrm{HF}$. In Tables 4-5 we present weight factors $\frac{n_{f A^{\prime}} \cdot n_{H F}}{n_{f A}}$ in fractional parentage decomposition, Eq.(3.7), which show the importance of the configuration in the given decay channel.

Table 4: Coefficients of fractional parentage for hyperhydrogen isotopes from ${ }_{\Lambda}^{9} \mathrm{Li}$

\begin{tabular}{|c|c|c|c|c|c|}
\hline$[f]_{A}$ & ${ }_{\Lambda}^{7} \mathrm{H}[321$ & $\mathrm{H}[32$ & $\mathrm{H}[3$ & $\mathrm{H}[3$ & $\mathrm{H}[2]$ \\
\hline${ }_{\Lambda}^{9} \mathrm{Li}[431]$ & $\frac{32}{70}$ & $\frac{30}{70}$ & $\frac{54}{70}$ & $\frac{15}{70}$ & $\frac{40}{70}$ \\
\hline [422] & $\frac{32}{56}$ & $\frac{15}{56}$ & $\frac{39}{56}$ & $\frac{10}{56}$ & $\frac{30}{56}$ \\
\hline$[4211]$ & $\frac{32}{90}$ & $\frac{15}{90}$ & $\frac{63}{90}$ & $\frac{15}{90}$ & $\frac{45}{90}$ \\
\hline$[332]$ & $\frac{32}{42}$ & $\frac{15}{42}$ & $\frac{21}{42}$ & $\frac{5}{42}$ & $\frac{21}{42}$ \\
\hline [3311] & $\frac{32}{56}$ & $\frac{15}{56}$ & $\frac{27}{56}$ & $\frac{6}{56}$ & $\frac{26}{56}$ \\
\hline$[3221]$ & $\frac{26}{70}$ & $\frac{10}{70}$ & $\frac{30}{70}$ & $\frac{5}{70}$ & $\frac{30}{70}$ \\
\hline [32111] & $\frac{10}{64}$ & $\frac{5}{64}$ & $\frac{39}{64}$ & $\frac{9}{64}$ & $\frac{24}{64}$ \\
\hline [41111] & - & - & - & $\frac{5}{35}$ & $\frac{15}{35}$ \\
\hline [2222] & - & - & - & - & $\frac{5}{14}$ \\
\hline
\end{tabular}

The TISM quantum numbers $[f],(\lambda \mu)$ allow us to select the most important basis wave functions. The source of hyperfragments are states $\Phi_{N}^{(A)}\left[f_{A}\right] \otimes \varphi_{0}^{\Lambda}$ with "destroyed" Young scheme $\left[f_{A}\right]\left(f_{1}<4\right)$. For illustration we give here the cfp for emission of ${ }_{\Lambda}^{4} \mathrm{H}$ hyperfragments:

$$
\begin{aligned}
& <\Phi_{N}^{(A)}[f](\lambda \mu) \varphi_{0}^{\Lambda} \mid \Phi_{N^{\prime}}^{(A-3)}\left[f^{\prime}\right](\lambda \mu)^{\prime} \cdot \varphi_{v}\left(R^{\prime}-R_{\mathrm{HF}}\right) \otimes \Phi_{0}^{(3)}[3] \varphi_{0}^{\Lambda}>=
\end{aligned}
$$

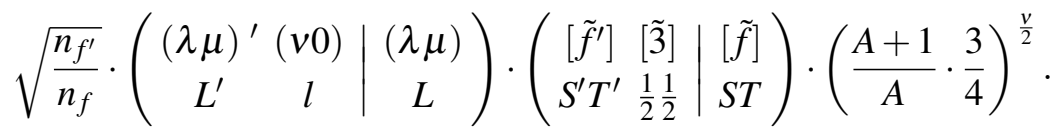

The last factor reflects the transformation of Jacobi coordinates.

In Table 5 we find the maximum probability of 1 for the decay

$$
{ }_{\Lambda}^{7} \mathrm{He} \rightarrow{ }_{\Lambda}^{6} \mathrm{H}+p
$$

from states with $\left[f_{6}\right]=[33]$.

Fig. 3 shows the shell model structures of ${ }^{7} \mathrm{Li},{ }_{\Lambda}^{7} \mathrm{He}^{*}$, and hyperfragments. The structure decomposition explains the forbidden decay of this state into He isotopes. 


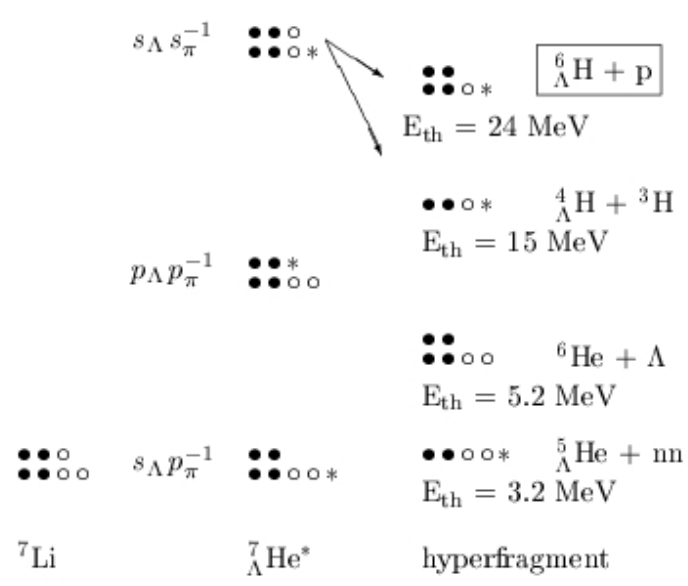

Figure 3: Shell model structure of ${ }^{7} \mathrm{Li},{ }_{\Lambda}^{7} \mathrm{He}^{*}$ and hyperfragments. The $\bullet, \circ, *$ mark neutron, proton, and $\Lambda$ respectively, at $s$-shell (first row) and at $p$-shell (second row). The consequences of the shell model structure of ${ }_{\Lambda}^{7} \mathrm{He}^{*}$ on its decay modes, i.e. the forbidden decay of this state to He isotopes, are illustrated.

This hyperhydrogen isotope ${ }_{\Lambda}^{6} \mathrm{H}$ is extremely interesting to study because it was claimed to be found by the FINUDA collaboration [20], but not seen in a recent J-PARC experiment [21]. A possible MAMI decay-pion experiment using a natural ${ }^{6,7} \mathrm{Li}$ target may give an independent answer on the existence of ${ }_{\Lambda}^{6} \mathrm{H}$. Lithium is the lightest of the considered target materials and it produces the lowest electromagnetic background in the experiment, so that it might be better suited for a search for heavy hyperhydrogen isotopes than the beryllium target used in the previous experiments.

\section{Suggestions and Outlook}

With the decay-pion spectroscopy a new chapter in hypernuclear physics can be opened and new hypernuclear isotopes can be produced.

The spectroscopy of monochromatic weak-decay pions can be used for the search of heavy hyperhydrogen isotopes [5]. The most interesting candidate is ${ }_{\Lambda}^{6} \mathrm{H}$. The three-particle decay of ${ }_{\Lambda}^{5} \mathrm{H}$ does not allow this isotope to be observed with this method, but observing ${ }_{\Lambda}^{7} \mathrm{H}$ seems to be possible.

Our primary task now is to incorporate $\mathrm{N}_{\min }+2$ configurations in the wave function of the target ground state and to include Strangeness Analogue States for estimation of the yields of hyperfragments:

$$
Y\left({ }_{\Lambda}^{6} \mathrm{H}\right): Y\left({ }_{\Lambda}^{3} \mathrm{H}\right): Y\left({ }_{\Lambda}^{4} \mathrm{H}\right) .
$$

Our approach is complementary to the transport model by Botvina et al. [22].

\section{Acknowledgement}

The authors thank J. Pochodzalla, L. Tang, and S.N. Nakamura for useful discussions throughout the work on this paper. O.M. and L.M. wish to thank organizers of XXII Baldin Seminar for their kind invitation. 
Table 5: Coefficients of fractional parentage for hyperhydrogen isotopes from ${ }_{\Lambda}^{7} \mathrm{He}$ and ${ }_{\Lambda}^{6} \mathrm{He}$

\begin{tabular}{|r|cccc|}
\hline$[f]_{A}$ & ${ }_{\Lambda}^{6} \mathrm{H}[32]$ & ${ }_{\Lambda}^{5} \mathrm{H}[31]$ & ${ }_{\Lambda}^{4} \mathrm{H}[3]$ \\
${ }_{\Lambda}^{3} \mathrm{H}[2]$ \\
\hline${ }_{\Lambda}^{7} \mathrm{He}[42]$ & $\frac{5}{9}$ & $\frac{6}{9}$ & $\frac{5}{9}$ & $\frac{6}{9}$ \\
{$[33]$} & $\frac{5}{5}$ & $\frac{3}{5}$ & $\frac{2}{5}$ & $\frac{3}{5}$ \\
{$[321]$} & $\frac{5}{16}$ & $\frac{6}{16}$ & $\frac{4}{16}$ & $\frac{8}{16}$ \\
{$[411]$} & - & $\frac{6}{10}$ & $\frac{2}{10}$ & $\frac{6}{10}$ \\
{$[3111]$} & - & $\frac{3}{10}$ & $\frac{2}{10}$ & $\frac{4}{10}$ \\
{$[222]$} & - & - & - & $\frac{2}{5}$ \\
{$[2211]$} & - & - & - & $\frac{3}{9}$ \\
\hline${ }_{\Lambda}^{6} \mathrm{He}[41]$ & - & $\frac{3}{4}$ & $\frac{2}{4}$ & $\frac{3}{4}$ \\
{$[32]$} & - & $\frac{3}{5}$ & $\frac{1}{5}$ & $\frac{3}{5}$ \\
{$[311]$} & - & $\frac{3}{6}$ & $\frac{1}{6}$ & $\frac{3}{6}$ \\
{$[221]$} & - & - & - & $\frac{2}{5}$ \\
{$[2111]$} & - & - & - & $\frac{1}{4}$ \\
\hline
\end{tabular}

\section{Appendix. TISM basis for $A=6,7$, and 9}

Components of the Strangeness Analogue States are marked bold.

Table 6: List of configurations $\Phi_{k}^{(5)} \otimes \varphi_{\ell}^{\Lambda}:\left[f_{5}\right](\lambda \mu)$

\begin{tabular}{|l|l|l|l|l|l|}
\hline \multicolumn{1}{|l|}{$\Phi_{1}^{(5)} \otimes \varphi_{0}^{\Lambda}$} & $(10)$ & \multicolumn{2}{|l|}{} \\
\cline { 1 - 4 }$\Phi_{1}^{(5)} \otimes \varphi_{1}^{\Lambda}$ & $(20)(01)$ & & \multicolumn{1}{|l}{} \\
$\Phi_{2}^{(5)} \otimes \varphi_{0}^{\Lambda}$ & $(20)$ & $(\mathbf{2 0})$ & $(01)$ & & \\
\hline$\Phi_{1}^{(5)} \otimes \varphi_{2}^{\Lambda}$ & $(30)(11)$ & & & & \\
$\Phi_{2}^{(5)} \otimes \varphi_{1}^{\Lambda}$ & $(30)(11)$ & $(30)(11)$ & $(11)(00)$ & & \\
$\Phi_{3}^{(5)} \otimes \varphi_{0}^{\Lambda}$ & $(30)^{2}(11)$ & $(30)(11)$ & $(30)(11)$ & $(11)$ & $(00)$ \\
\hline$\left[f_{5}\right]:$ & {$[41]$} & {$[32]$} & {$[311]$} & {$[221]$} & {$[2111]$} \\
\hline
\end{tabular}


Table 7: List of configurations $\Phi_{k}^{(6)} \otimes \varphi_{\ell}^{\Lambda}:\left[f_{6}\right](\lambda \mu)$

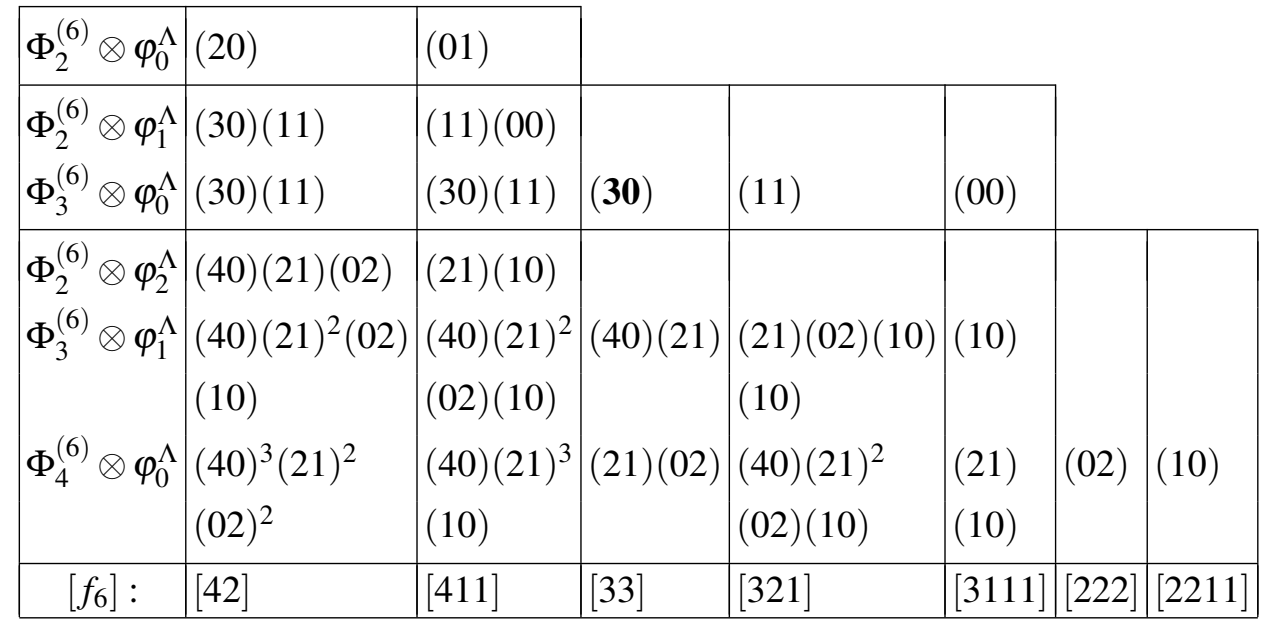

Table 8: List of configurations $\Phi_{k}^{(8)} \otimes \varphi_{\ell}^{\Lambda}:\left[f_{8}\right](\lambda \mu)$, the first part.

\begin{tabular}{|l|l|l|l|l|}
\hline$\Phi_{4}^{(8)} \otimes \varphi_{0}^{\Lambda}$ & $(40)$ & $(21)$ & $(02)$ & $(10)$ \\
\hline$\Phi_{4}^{(8)} \otimes \varphi_{1}^{\Lambda}$ & $(50)(31)$ & $(31)(12)(20)$ & $(12)(01)$ & $(20)(01)$ \\
$\Phi_{5}^{(8)} \otimes \mathbf{S}_{\Lambda}$ & $(31)$ & $(50)(31)^{2}(20)(01)$ & $(31)(12)(20)(01)$ & $(31)(12)^{2}(20)^{2}(01)$ \\
\hline$\Phi_{4}^{(8)} \otimes \varphi_{2}^{\Lambda}$ & $(60)(41)(22)$ & $(41)(22)(03)(30)(11)$ & $(22)(11)(00)$ & \\
$\Phi_{5}^{(8)} \otimes \varphi_{1}^{\Lambda}$ & $(41)(22)(30)$ & $\begin{array}{l}(60)(41)^{3}(22)^{2}(03) \\
(60)(41)^{3}(22)^{2}(03)\end{array}$ & $\begin{array}{l}(41)(22)^{3}(03)^{2} \\
(30)^{2}(11)^{3}(00)\end{array}$ & $(30)^{3}(11)^{4}(00)$ \\
$(81)^{2}(00)$ & $(41)^{3}(22)(03)$ \\
$\Phi_{6}^{(8)} \otimes \varphi_{0}^{\Lambda}$ & $(60)^{2}(41)^{2}(22)^{3}$ & $(60)^{2}(41)^{5}(22)^{5}(03)^{5}$ & $(60)(41)^{2}(22)^{4}$ \\
$(03)^{2}(11)^{2}(00)$ & $(30)^{4}(11)^{7}(00)$ & $(30)^{2}(11)^{4}(00)^{2}$ & $(30)^{5}(11)^{4}$ \\
\hline$\left[f_{8}\right]:$ & {$[44]$} & {$[431]$} & {$[422]$} & {$[4211]$} \\
\hline
\end{tabular}

Table 9: List of configurations $\Phi_{k}^{(8)} \otimes \varphi_{\ell}^{\Lambda}:\left[f_{8}\right](\lambda \mu)$, the second part.

\begin{tabular}{|l|l|l|l|l|l|l|}
\hline$\Phi_{5}^{(8)} \otimes \varphi_{0}^{\Lambda}$ & $(20)$ & $(12)$ & $(20)$ & \multicolumn{2}{|l|}{$(01)$} & \\
\cline { 1 - 4 }$\Phi_{5}^{(8)} \otimes \varphi_{1}^{\Lambda}$ & $(30)(11)$ & $(22)(03)(11)$ & $(30)(11)$ & $(11)(00)$ & & \\
$\Phi_{6}^{(8)} \otimes \varphi_{0}^{\Lambda}$ & $(22)$ & $(41)(03)$ & $(41)(22)(03)$ & $(30)(11)$ & $(30)(11)$ & $(00)$ \\
& $(30)^{2}(11)^{2}$ & $(30)(11)^{2}(00)$ & $(30)(11)^{2}(00)$ & & & \\
\hline$\left[f_{8}\right]:$ & {$[41111]$} & {$[332]$} & {$[3311]$} & {$[3221]$} & {$[32111]$} & {$[2222]$} \\
\hline
\end{tabular}




\section{References}

[1] V. Kekelidze et al., these Proceedings;

V.A. Kireyeu, these Proceedings.

[2] O. Majlingova, L. Majling, PoS (Baldin ISHEEP XXI) 101, (2012).

[3] J. Pochodzalla, Acta Phys. Polon. B 42, 833 (2011).

[4] P. Achenbach et al. (A1 Collaboration), Nucl. Phys. A 881, 187 (2012);

A. Esser et al. (A1 Collaboration): Observation of ${ }_{\Lambda}^{4} \mathrm{H}$ hyperhydrogen by decay-pion spectroscopy in electron scattering. arXiv:1501.06823.

[5] R.H. Dalitz, Nucl. Phys. A 754, 14c (2005).

[6] O. Hashimoto, H. Tamura, Progr. Part. Nucl. Phys. 57, 564 (2006).

[7] F. Cusanno et al., Phys. Rev. Lett. 103, 202501 (2009).

[8] A. Gal, J.M. Soper, R.H. Dalitz, Ann. Phys. (NY) 63, 53 (1971); 72, 445 (1972); 113, 79 (1978).

[9] D. J. Millener, in Topics in Strangeness Nuclear Physics, Lecture Notes in Physics, Vol. 724, eds. P. Bydžovský, A. Gal, and J. Mareš (Springer, New York, 2007), p. 31.

[10] R. Bertini et al., Nucl. Phys. A 368, 365 (1981).

[11] H.J. Lipkin, Phys. Rev. Lett. 14, 18 (1965).

[12] L. Majling et al., Phys. Lett. B 92, 256 (1980).

[13] J. Žofka et al., Phys. Part. Nucl. 22 (1991); 28, 101 (1997).

[14] L. Majling, Nucl. Phys. A 639, 125c (1998).

[15] G.M. Urcioli et al.: Spectroscopy of ${ }_{\Lambda}^{9} \mathrm{Li}$ by electroproduction. arXiv: 1405.5839.

[16] C.W. Johnson: Spin-orbit decomposition of ab initio wave functions. arXiv: 1409.7355.

[17] J.P. Elliott, Proc. Roy. Soc. A 245, 128; 562 (1958).

[18] V. Kukulin, Yu. Smirnov, L. Majling, Nucl. Phys. A 103, 681 (1967).

[19] R.V. Jolos, L. Majling, O. Majlingova, in preparation.

[20] M. Agnello et al., Nucl. Phys. A 881, 269 (2012).

[21] H. Sugimura et al., Phys. Lett. B 729, 39 (2014).

[22] A.S. Botvina et al., Nucl. Phys. A 881, 228 (2012);

A.S. Botvina et al., arXiv:1412.6665. 